\title{
TU/e EmonOWEN

\section{Perturbation bounds for root-clustering of linear systems in a specified second order subregion}

\section{Citation for published version (APA):}

Bakker, W., Luo, J. S., \& Johnson, A. (1995). Perturbation bounds for root-clustering of linear systems in a specified second order subregion. IEEE Transactions on Automatic Control, 40, 473-478.

https://doi.org/10.1109/9.376060

DOI:

10.1109/9.376060

Document status and date:

Published: 01/01/1995

\section{Document Version:}

Publisher's PDF, also known as Version of Record (includes final page, issue and volume numbers)

\section{Please check the document version of this publication:}

- A submitted manuscript is the version of the article upon submission and before peer-review. There can be important differences between the submitted version and the official published version of record. People interested in the research are advised to contact the author for the final version of the publication, or visit the $\mathrm{DOI}$ to the publisher's website.

- The final author version and the galley proof are versions of the publication after peer review.

- The final published version features the final layout of the paper including the volume, issue and page numbers.

Link to publication

\section{General rights}

Copyright and moral rights for the publications made accessible in the public portal are retained by the authors and/or other copyright owners and it is a condition of accessing publications that users recognise and abide by the legal requirements associated with these rights.

- Users may download and print one copy of any publication from the public portal for the purpose of private study or research.

- You may not further distribute the material or use it for any profit-making activity or commercial gain

- You may freely distribute the URL identifying the publication in the public portal.

If the publication is distributed under the terms of Article 25fa of the Dutch Copyright Act, indicated by the "Taverne" license above, please follow below link for the End User Agreement:

www.tue.nl/taverne

Take down policy

If you believe that this document breaches copyright please contact us at:

openaccess@tue.nl

providing details and we will investigate your claim. 


\section{Technical Notes and Correspondence}

\section{Perturbation Bounds for Root-Clustering of Linear Systems in a Specified Second Order Subregion}

\author{
W. Bakker, J. S. Luo, and A. Johnson
}

is given, followed by the "shifting theorem." In Section IV, bounds for structured uncertainties are given. For both cases, the bounds can be improved by optimization programs. In Section V, the results are illustrated with examples, followed by two general remarks. We end the paper with conclusions in Section VI.

\section{Generalized Lyapunov RoOt-Clustering Theory}

Let the perturbed continuous-time system be represented by

$$
\dot{\mathbf{x}}(t)=(A+E) \mathbf{x}(t)
$$

and let the perturbed discrete-time system be represented by

$$
\mathbf{x}(k+1)=(A+E) \mathbf{x}(k)
$$

We study the problem of how to guarantee the location of the eigenvalues of a perturbed system matrix in a specified (symmetric) second-order subregion of the complex plane. The perturbation may be structured or unstructured. The subregions discussed are, in the continuous-time case, subregions of the left-half complex plane (LHP) and in the discrete-time case subregions of the unit disk (UD) centered at the origin. Recently, Abdul-Wahab [1] and [2] discussed second-order subregions for discrete-time and continuous time systems, but the results have turned out to be erroneous. The errors have been pointed out by Yedavalli [3] for the continuoustime case and by Bakker and Luo [4] for the discrete-time case More recently, Yedavalli [5] obtained results for first and secondorder subregions using Generalized Lyapunov Theory presented by Gutman and Jury [6].

Conservatism of the paper of Yedavalli [5] is reduced in severa ways. First, the norms used to compute the bound are taken at a later stage compared to the bounds of Yedavalli. Next, an important result is, for unstructured uncertainties, that the bound can be improved for subregions with center or focus at $(\alpha, 0)$. This improved bound is obtained by shifting the center or focus of the subregion along the real axis to the origin and computing the bound for the "shifted eigenvalue" matrix. Generally, a large $\alpha$ yields a large improvement. Finally, from Luo et al. [7] we are motivated to use the square root of positive definite matrix $Q\left(=S^{T} S\right)$ in the Lyapunov equations; the result can be improved by choosing the $S$-matrix appropriately. Using a personal computer, the optimal $S$-matrix can be found by an optimization program in Matlab (e.g., fminu.m [9]).

This paper is organized as follows. In Section II the robust stability analysis for state-space models of continuous- and discrete-time systems using the Generalized Lyapunov Theory [6] is described. In Section III we introduce bounds for both continuous and discrete-time systems with unstructured uncertainties. A motivation for "shifting"

Manuscript received April 19, 1993; revised January 27, 1994 and May $18,1994$.

W. Bakker is with the ASPC-group, Turnkiek Process Control, Henry Dunantstraat 38, 3822 XE Amersfoort, The Netherlands.

J. S. Luo is with Process Dynamics and Control, Kramers Laboratory, Delft University of Technology, Prins Bernhardlaan 6, 2628 BW Delft, The Netherlands.

A. Johnson passed away in February, 1994; he was head of the section Process Dynamics and Control at the Kramers Laboratory.

IEEE Log Number 9407237. where the state vector is $\mathbf{x}(t)$ or $\mathbf{x}(k) \in \mathbb{R}^{n}$, the time-invariant system matrix $A \in \mathbb{R}^{n \times n}$ and the perturbation matrix $E \in \mathbb{R}^{n \times n}$. If the structure of the perturbation is known, the substitution

$$
E=\sum_{i=1}^{m} \epsilon_{i} E_{i}
$$

is made. Here $\epsilon_{i}(i=1,2, \cdots, m)$ are time-invariant uncertain parameters which are assumed to lie in the interval around zero $\left(\epsilon_{i} \in[-\epsilon, \epsilon]\right.$ where $\left.\epsilon>0\right)$ and $E_{i}(i=1,2, \cdots, m)$ are constant matrices determined by the structure of the parameter uncertainties.

Any symmetric second order subregion in the complex plane ( $(s$ or $z)=x+i y)$ is described by $(x, y)$ satisfying the inequality [6]

where $\gamma_{f g}$ is a real coefficient (note the difference with the boldfaced state vector $\mathrm{x}$ and the real coordinate $x$ ). It is clear that this region is symmetrical with respect to the $x$ axis. This inequality describes for example circles, ellipses, left parabolas, etc. Let us now define, as in [6]

$$
\begin{aligned}
c_{00}=\gamma_{00}, \quad c_{10}=c_{01}=\frac{1}{2} \gamma_{10}, \quad c_{11} & =\frac{1}{2}\left(\gamma_{20}+\gamma_{02}\right), \\
c_{20} & =c_{02}=\frac{1}{4}\left(\gamma_{20}-\gamma_{02}\right)
\end{aligned}
$$

so we can present the Lyapunov criterion for root-clustering in a specified second-order subregion $\Omega_{2}$ [6]: Let $\gamma_{20}+\gamma_{02} \geq 0$ in (2.3) and let the coefficients $c_{p q}$ be given by (2.4). If and only if for a given positive definite Hermitian matrix $Q$ the following generalized Lyapunov equation (GLE)

$$
\begin{array}{r}
c_{00} P+c_{10}\left(A^{T} P+P A\right)+c_{11} A^{T} P A+c_{20}\left(\left(A^{T}\right)^{2} P+P A^{2}\right) \\
=-Q
\end{array}
$$

has a positive definite solution $P$, then all the eigenvalues of the nonperturbed system $[E=0$ in (2.1)] are located inside the defined subregion (2.3). Equation (2.5) can be solved using the Kronecker product; for a detailed description, see [6].

$$
\Omega_{2}=\left\{(x, y): \gamma_{00}+\gamma_{10} x+\gamma_{20} x^{2}+\gamma_{02} y^{2}<0\right\}
$$




\section{Perturbation Bounds For Unstructured UnCERTAinties}

In this section new stability robustness bounds for systems with unstructured uncertainties based on the Generalized Lyapunov RootClustering Theory presented in Section II are given. It is shown that "shifting," for a large class of subregions, yields improved bounds. These results are organized in the "shifting theorem."

In the next theorem we give less conservative bounds on the norm of the perturbation matrix $E$ so that the eigenvalues are located in the specified subregion. There is no knowledge assumed about the structure of the perturbation.

Theorem 3.1: Suppose

1) The perturbed system is described by (2.1).

2) The eigenvalues of the nonperturbed system matrix $A$ are located inside a subregion $\Omega_{2}$ defined by (2.3).

3) $S^{T}$ and $S$ are full rank (invertible) matrices satisfying $Q=$ $S^{T} S$.

4) $P$ is a symmetric positive definite matrix defined by (2.5).

Then the perturbed system matrix $A+E$ has all the eigenvalues located in the subregion $\Omega_{2}$ described by (2.3), if

$$
\|E\|<\mu=\frac{b}{2 a}\left(-1+\sqrt{1+\frac{4 a}{b^{2}}}\right)
$$

with

$$
\begin{aligned}
a \equiv & \left|c_{11}\right|\|P\|\left\|S^{-1}\right\|^{2}+2\left|c_{20}\right|\left\|S^{-1}\right\|\left\|P S^{-1}\right\| \\
b \equiv & 2\left|c_{10}\right|\left\|S^{-1}\right\|\left\|P S^{-1}\right\|+2\left|c_{11}\right|\left\|S^{-1}\right\|\left\|P A S^{-1}\right\| \\
& +2\left|c_{20}\right|\left\|S^{-T} A^{T}\right\|\left\|P S^{-1}\right\| \\
& +2\left|c_{20}\right|\left\|S^{-1}\right\|\left\|A^{T} P S^{-1}\right\|
\end{aligned}
$$

and where $\|A\|=$ the spectral norm of the matrix $A$ (the largest singular value of $A$ ).

Proof: See Appendix.

Remark 3.1: For first-order subregions, $c_{11}=c_{20}=0$ (2.3) and (2.4). In that case the upper bound (3.1) is obtained from (A.2) with $a=0$ to be $\mu=1 / b$.

\section{Shifting of Subregions}

The bound $\mu$ depends on the matrices $A, P$, and $S$ and on the coefficients that describe the subregion. Consider the role of the system matrix in the bound (summarized in Fact 1). Of interest are subregions of the form

$$
\Omega_{2}=\left\{(x, y): \quad((x-\alpha) / v)^{f}+(y / w)^{g}-u<0\right\}
$$

where $(\alpha, v, w, u) \in \mathbb{R}$ and $v>0, w>0 ; f=1,2$, and $g=0,2$. Examples are a circle, a left-parabola, an ellipse, a vertical strip, etc. The eigenvalues of system matrix $A$ have to be located in this subregion. Next, for convenience, restrict the subregion to be a circular subregion of the LHP, with center $(\alpha, 0)$ and radius 1 satisfying $\alpha<-1, f=2=g, u=1=v=w$ in (3.2) (for other subregions similar arguments hold). Then the eigenvalues of the matrix $A$ have to be located within a distance 1 from $(\alpha, 0)$ and therefore satisfy

$$
(\alpha-1)<\operatorname{Re}(\lambda(A))<(\alpha+1) .
$$

Because $\alpha<-1 \Leftrightarrow \alpha+1<0$, taking norms

$$
|\operatorname{Re}(\lambda(A))|>|\alpha+1| \text {. }
$$

From [20]

$$
\begin{aligned}
\|A\| & \geq|\lambda(A)|=\left(\operatorname{Re}(\lambda(A))^{2}+\operatorname{Im}(\lambda(A))^{2}\right)^{1 / 2} \\
& \geq|\operatorname{Re}(\lambda(A))|>|\alpha+1| .
\end{aligned}
$$

Fact 1: The coefficients $c_{11}$ and $c_{20}$ and the solution for $P$ (2.5) (see Remark 3.2), used in the bound (3.1a), are not affected by shifting.

Fact 2: From (3.3), $\|A\|>|\alpha+1|$. This implies, with Fact 1, that when $S=I$ at least the third term in (3.1b) is multiplied by a factor larger than $|\alpha+1|$. Thus when $\alpha$ is small $(\alpha \ll-1),|\alpha+1|$ is large and consequently that $\|A\|$ is large. So a large $\alpha$ causes a large $b$. For subregions as a left-parabola or a vertical strip, with center or focus at $(\alpha, 0)$, similar inequalities hold.

Fact 3: The value of $a$ is unaffected by shifting (follows from Fact 1).

Fact 4: When $b$ becomes large ( $a$ unaffected) then the bound becomes small (i.e., very conservative). This is even more obvious when, with $b \gg a$, a Taylor series expansion is used for the square root in (3.1): $\mu \approx 1 / b$.

We have shown that the bound is conservative when subregions with center or focus at $(\alpha, 0), \alpha$ sufficiently large, are considered. In the next theorem, it is shown that it is possible to shift the subregions (3.2) from $(\alpha, 0)$ to $(0,0)$ and to compute the bound for the "shifted eigenvalue" system matrix with Theorem 3.1. With the facts are given above, the improvement of the bound should be clear.

Theorem 3.2 "Shifting Theorem:" Root-clustering in a subregion $\Omega_{2}$ (3.2) of the complex plane for a system matrix $A$ is equivalent to root-clustering in a shifted subregion $\tilde{\Omega}_{2}$ (3.4) of the complex plane for a "shifted eigenvalue" system matrix $\tilde{A}$, where

$$
\begin{gathered}
\tilde{\Omega}_{2}=\left\{(\tilde{x}, y):(\tilde{x} / v)^{f}+(y / w)^{g}-u<0\right\} \\
\tilde{x}=x-\alpha \\
\tilde{A}=A-\alpha I .
\end{gathered}
$$

Proof: Using (3.4c)

$$
\lambda(A+E)=\lambda(\tilde{A}+\alpha I+E)=\lambda(\tilde{A}+E)+\alpha
$$

and for the real coordinate of the subregion, with (3.4b)

$$
x=\tilde{x}+\alpha .
$$

Thus, the bound on the perturbation $E$ can be computed for the shifted subregion and "shifted eigenvalue" system matrix, being valid for the original subregion and system matrix.

Remark 3.2: Theorem 3.2 can also be proved using the General Lyapunov Equation; that is, when (3.4c) is substituted in the GLE of the shifted subregion (with coefficients (2.4) for this shifted subregion) after some standard manipulations it can be shown that for subregions that can be shifted the solution $P$ of the GLE of the shifted subregion equals the solution $P$ of the GLE of the original subregion.

Remark 3.3: For convenience and motivated by fact that the coefficient $c_{10}$ becomes zero in the bound (see (3.1b) and Fact 4), the subregion is shifted along the real axis to the origin. For some very specific cases, the best bound is not obtained by shifting exactly to the origin. In that case, one can find the "optimal shift" along the real axis which yields the best bound.

Remark 3.4: The result (3.1) is a function of $S$. In [7] it is shown that for similar cases an optimization (maximization) of $\mu(a(S), b(S))$ reduces conversatism. This can be done with the program fminu.m in Matlab [9]. The power of this method will be illustrated with examples in Section V.

Table I shows the results of this section for some second order subregions. When the regions satisfy (3.2), the parameters holding for the shifted subregions are presented. 
TABLE I

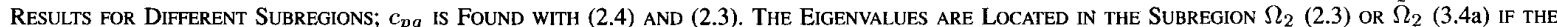
Condition in Theorem 3.1 with a and b as Below is Satisfied. The Equations After the " " are Obtained from the Shifted Subregion (Theorem 3.2)

\begin{tabular}{|c|c|c|c|c|c|c|c|}
\hline Subregion & $\Omega_{2}=(x, y):$ & $c_{00}$ & $c_{10}$ & $c_{11}$ & $c_{20}$ & $\mathrm{a}$ & b \\
\hline circle - & $(x / r)^{2}+(y / r)^{2}-1<0$ & -1 & 0 & $r^{-2}$ & 0 & $\left|\mathrm{r}^{-2}\right|\|\mathrm{P}\|\left\|\mathrm{S}^{-1}\right\|^{2}$ & $2\left|\mathbf{r}^{-2}\right|\left\|S^{-1}\right\|\left\|\mathrm{PAS}^{-1}\right\|$ \\
\hline parabola & $4 p x+y^{2}<0$ & 0 & $2 p$ & $\frac{1}{2}$ & $-\frac{1}{4}$ & $\left\{\begin{array}{r}\frac{1}{2}\|P\|\left\|S^{-1}\right\|^{2} \\
+\frac{1}{2}\left\|S^{-1}\right\|\left\|S^{-1}\right\|\end{array}\right.$ & $\begin{array}{l}4 p\left\|S^{-1}\right\|\left\|S^{-1}\right\|+\left\|S^{-1}\right\|\left\|P A S^{-1}\right\| \\
+\frac{1}{2}\left\|S^{-T} A^{T}\right\|\left\|S^{-1}\right\|+\frac{1}{2}\left\|S^{-1}\right\|\left\|A^{T} P^{-1}\right\|\end{array}$ \\
\hline ellipse & $(x / v)^{2}+(y / w)^{2}-1<0$ & -1 & 0 & $\frac{1}{2}\left(v^{-2}+w^{-2}\right)$ & $\frac{1}{4}\left(v^{-2}-w^{-2}\right)$ & $\left.\mid \begin{array}{l}\frac{1}{2}\left(v^{-2}+w^{-2}\right)\|P\|\left\|S^{-1}\right\|^{2} \\
+\frac{1}{2}\left(v^{-2}-w^{-2}\right)\left\|S^{-1}\right\|\left\|P S^{-1}\right\|\end{array}\right]$ & $\begin{array}{l}\left(\mathrm{v}^{-2}+w^{-2}\right)\left\|\mathrm{S}^{-1}\right\|\left\|\mathrm{PAS}^{-1}\right\| \\
+\frac{1}{2}\left|\mathrm{v}^{-2}-\mathrm{w}^{-2}\right|\left(\left\|\mathrm{S}^{-\mathrm{T}} \mathrm{A}^{\mathrm{T}}\right\|\left\|\mathrm{PS}^{-1}\right\|+\left\|\mathrm{S}^{-1}\right\|\left\|\mathrm{A}^{\mathrm{T}} \mathrm{PS}^{-1}\right\|\right)\end{array}$ \\
\hline $\begin{array}{l}\text { Vertical } \\
\text { strip }\end{array}$ & $\mathrm{x}^{2}-\beta^{2}<0$ & $-\beta^{2}$ & 0 & $\frac{1}{2}$ & $\frac{1}{4}$ & $\mid \begin{array}{l}\frac{1}{2}\|P\|\left\|S^{-1}\right\|^{2} \\
+\frac{1}{2}\left\|S^{-1}\right\|\left\|S^{-1}\right\|\end{array}$ & $\begin{array}{l}\left\|S^{-1}\right\|\left\|\mathrm{PAS}^{-1}\right\|+\frac{1}{2}\left\|\mathrm{~S}^{-\mathrm{T}} \mathrm{A}^{\mathrm{T}}\right\|\left\|\mathrm{PS}^{-1}\right\| \\
+\frac{1}{2}\left\|\mathrm{~S}^{-1}\right\|\left\|\mathrm{A}^{\mathrm{T}} \mathrm{PS}^{-1}\right\|\end{array}$ \\
\hline $\begin{array}{l}\text { Ride } \\
\text { quality } \\
\text { [6] }\end{array}$ & $\begin{array}{l}\text { 1. }-\mathrm{x}^{2}-\mathrm{y}^{2}+\rho_{1}^{2}<0 \\
\text { 2. } \mathrm{x}^{2}+\mathrm{y}^{2}-\rho_{2}^{2}<0 \\
\text { 3. }-\omega^{2}+\mathrm{y}^{2}<0\end{array}$ & \begin{tabular}{c|}
$\rho_{1}^{2}$ \\
$-\rho_{2}^{2}$ \\
$-\omega^{2}$
\end{tabular} & $\begin{array}{l}0 \\
0 \\
0\end{array}$ & $\begin{array}{l}-1 \\
1 \\
\frac{1}{2}\end{array}$ & $\begin{array}{l}0 \\
0 \\
-\frac{1}{4}\end{array}$ & $\begin{array}{l}\|P\|\left\|S^{-1}\right\|^{2} \\
\|P\|\left\|S^{-1}\right\|^{2} \\
\frac{1}{2}\|P\|\left\|S^{-1}\right\|^{2}+\frac{1}{2}\left\|S^{-1}\right\|\left\|P S^{-1}\right\|\end{array}$ & $\begin{array}{l}2\left\|S^{-1}\right\|\left\|P S^{-1}\right\| \\
2\left\|S^{-1}\right\|\left\|P^{-1}\right\| \\
\left\|S^{-1}\right\|\left\|P^{-1}\right\| S^{-1}\left\|+\frac{1}{2}\right\| S^{-T} A^{T}\|\| P^{-1}\left\|+\frac{1}{2}\right\| S^{-1}\|\| A^{T} P^{-1} \|\end{array}$ \\
\hline
\end{tabular}

\section{Perturbation Bounds for STRUCTUREd UnCERTAinties}

For structured uncertainties it is possible to obtain less conservative results. In the next theorem we obtain a perturbation bound for this case.

Theorem 4.1: The same assumptions as in Theorem 3.1 1)-4) are made. The system is given by (2.1) with the perturbation as in (2.2). The eigenvalues of the perturbed system are located in the described subregion $(2.3)$, if

$$
|\epsilon|<\mu=\frac{b}{2 a}\left(-1+\sqrt{1+\frac{4 a}{b^{2}}}\right)
$$

where

$$
\begin{gathered}
a \equiv\left\|\sum_{i=1}^{m} \sum_{j=i}^{m}\left|P_{i j}^{*}\right|\right\|, \quad b \equiv\left\|\sum_{i=1}^{m}\left|P_{i}^{*}\right|\right\| \\
P_{i j}^{*} \equiv S^{-T} P_{i j} S^{-1}, \quad P_{i}^{*} \equiv S^{-T} P_{i} S^{-1} \\
P_{i j} \equiv c_{11}\left(E_{i}^{T} P E_{j}+E_{j}^{T} P E_{i}\right) \\
+c_{20}\left(\left(E_{i} E_{j}\right)^{T} P+P E_{i} E_{j}+\left(E_{j} E_{i}\right)^{T} P+P E_{j} E_{i}\right) \\
P_{i} \equiv c_{10}\left(E_{i}^{T} P+P E_{i}\right)+c_{11}\left(E_{i}^{T} P A+A^{T} P E_{i}\right) \\
+c_{20}\left(\left(E_{i} A\right)^{T} P+P E_{i} A+\left(A E_{i}\right)^{T} P+P A E_{i}\right)
\end{gathered}
$$

and where $|A|=\left\{\left|a_{i j}\right|\right\}$, with $A=\left\{a_{i j}\right\}$, a $n \times n$ matrix.

Proof: See Appendix.

Remark 4.1: For first order subregions, (4.1) becomes $\mu=1 / b$ (see Remark 3.1).

Remark 4.2: Optimization of $\mu(a(S), b(S))$ in (4.1) reduces conservatism (see Remark 3.4).

Similar to Section III, it is possible to work out this general result for different subregions. This is done in Table II.

\section{EXAmPles for Different SeCOND ORdER Subregions}

\section{Root-Clustering in a Circle with Unstructured Uncertainties}

The result will now be illustrated with an example. We take the same plant matrix as Yedavalli [5]

$$
A=\left[\begin{array}{rr}
-4.3 & -0.4 \\
0.2 & -3.4
\end{array}\right]
$$

with eigenvalues $\lambda_{1}=-4.2, \lambda_{2}=-3.5$. The circular subregion is defined with $\alpha=-4, v=w=1, u=1, f=g=2$ in (3.2). First, taking $Q=S=I$, (3.1) gives the upper perturbation bound

$$
\mu_{c, S=I}=0.0370
$$

where the result in [5] is $\mu_{c, y \in d}=0.0341$. This is an improvement of $8.5 \%$.

Secondly, we shift the center of circle to the origin (Theorem 3.2) and apply our root-clustering Theorem 3.1 for the system matrix $\tilde{A}$ in the subregion $\tilde{\Omega}_{2}(3.4 \mathrm{a})$. This gives

$$
\tilde{A}=\left[\begin{array}{rr}
-0.3 & -0.4 \\
0.2 & 0.6
\end{array}\right]
$$

with eigenvalues $\lambda_{1}(\tilde{A})=-0.2, \lambda_{2}(\tilde{A})=0.5$. The upper bound on $\|E\|$ becomes

$$
\tilde{\mu}_{c, S=I}=0.3782 \text {. }
$$

Comparing the result $\tilde{\mu}_{c, S=I}$ to $\mu_{c, y \in d}$ [5] we see that the bound is improved by $1009 \%$.

Thirdly, for the shifted subregion $\tilde{\Omega}_{2}$ and the "shifted eigenvalue" matrix $\tilde{A}$, optimizing $S$ gives the solutions, with (2.5)

$$
S=\left[\begin{array}{ll}
0.9013 & 0.0723 \\
0.0760 & 0.8494
\end{array}\right], \quad P=\left[\begin{array}{ll}
0.9098 & 0.2981 \\
0.2981 & 1.1395
\end{array}\right]
$$

With (3.1) this gives

$$
\tilde{\mu}_{c, S=S_{o p t}}=0.3865 \text {. }
$$

Comparing $\tilde{\mu}_{c, s=S_{\text {opt }}}$ and $\tilde{\mu}_{c, S=I}$ an improvement of $2.2 \%$ is achieved. 
TABLE II

Results for Different Subregions; $c_{p q}$ IS Found with (2.4) and (2.3). The Eeigenvalues are Located in the Subregion $\Omega_{2}$ If the Conditions in Theorem 4.1 with $P_{i j}$ and $P_{i}$ as Below are Satisfied

\begin{tabular}{|c|c|c|c|c|c|c|c|}
\hline Subregion & $\Omega_{2}=(x, y):$ & $c_{00}$ & $\mathrm{c}_{10}$ & $c_{11}$ & $c_{20}$ & $P_{i j}$ & $P_{i}$ \\
\hline circle & $(x-\alpha)^{2}+y^{2}-r^{2}<0$ & $\alpha^{2}-r^{2}$ & $-\alpha$ & 1 & 0 & $E_{i}^{T} P E_{j}+E_{j}^{T} P E_{i}$ & $-\alpha\left(E_{i}^{T} P+P E_{i}\right)+\left(E_{i}^{T} P A+A^{T} P E_{i}\right)$ \\
\hline parabola & $-4 p \alpha+4 p x+y^{2}<0$ & $-4 p \alpha$ & $2 p$ & $\frac{1}{2}$ & $-\frac{1}{4}$ & $\left\{\begin{array}{l}\frac{1}{2}\left(E_{i}^{T} P E_{j}+E_{j}^{T} P E_{i}\right) \\
-\frac{1}{4}\left(E_{j}^{T} E_{i}^{T} P+P E_{i} E_{j}+E_{i}^{T} E_{j}^{T} P+P E_{j} E_{i}\right)\end{array}\right.$ & $\begin{array}{l}2 p\left(E_{i}^{T} P+P E_{i}\right)+\frac{1}{2}\left(E_{i}^{T} P A+A^{T} P E_{i}\right) \\
-\frac{1}{4}\left(A^{T} E_{i}^{T} P+P E_{i} A+E_{i}^{T} A^{T} P+P A E_{i}\right)\end{array}$ \\
\hline ellipse & $\begin{array}{l}((x-\alpha) / v)^{2} \\
+(y / w)^{2}-1<0\end{array}$ & $(\alpha / v)^{2}-1$ & $-\alpha v^{-2}$ & $2 \frac{1}{2}\left(v^{-2}+w^{-2}\right)$ & $\frac{1}{4}\left(v^{-2}-w^{-2}\right)$ & $\left\{\begin{array}{l}\frac{1}{2}\left(v^{-2}+w^{-2}\right)\left(E_{j}^{T} P E_{j}+E_{j}^{T} P E_{i}\right) \\
+\frac{1}{4}\left(v^{-2}-w^{-2}\right)\left(E_{j}^{T} E_{i}^{T} P+P E_{i} E_{j}+E_{i}^{T} E_{j}^{T} P+P E_{j} E_{i}\right)\end{array}\right.$ & $\begin{array}{l}-\alpha v^{-2}\left(E_{i}^{T} P+P E_{i}\right)+\frac{1}{2}\left(v^{-2}+w^{-2}\right)\left(E_{i}^{T} P A+A^{T} P E_{i}\right) \\
+\frac{1}{4}\left(v^{-2}-w^{-2}\right)\left(A^{T} E_{i}^{T} P+P E_{i} A+E_{i}^{T} A^{T} P+P A E_{i}\right)\end{array}$ \\
\hline $\begin{array}{r}\text { Vertical } \\
\text { strip }\end{array}$ & $(x-\alpha)^{2}-\beta^{2}<0$ & $\alpha^{2}-\beta^{2}$ & $-\alpha$ & $\frac{1}{2}$ & $\frac{1}{4}$ & $\begin{array}{l}\frac{1}{2}\left(E_{i}^{T} P E_{j}+E_{j}^{T} P E_{i}\right) \\
+\frac{1}{4}\left(E_{j}^{T} E_{i}^{T} P+P E_{i} E_{j}+E_{i}^{T} E_{j}^{T} P+P E_{j} E_{i}\right)\end{array}$ & $\begin{array}{l}-\alpha\left(E_{i}^{T} P+P E_{i}\right)+\frac{1}{2}\left(E_{i}^{T} P A+A^{T} P E_{i}\right) \\
+\frac{1}{4}\left(A^{T} E_{i}^{T} P+P E_{i} A+E_{i}^{T} A^{T} P+P A E_{i}\right)\end{array}$ \\
\hline $\begin{array}{l}\text { Ride } \\
\text { quality } \\
{[6]}\end{array}$ & $\begin{array}{l}\text { 1. } \rho_{1}^{2}<\mathrm{x}^{2}+\mathrm{y}^{2} \\
\text { 2. } \mathrm{x}^{2}+\mathrm{y}^{2}<\rho_{2}^{2} \\
\text { 3. }-\omega^{2}+\mathrm{y}^{2}<0\end{array}$ & $\begin{array}{l}\rho_{1}^{2} \\
-\rho_{2}^{2} \\
-\omega^{2}\end{array}$ & $\mid \begin{array}{l}0 \\
0 \\
0\end{array}$ & $\mid \begin{array}{l}-1 \\
1 \\
\frac{1}{2}\end{array}$ & $\begin{array}{l}0 \\
0 \\
-\frac{1}{4}\end{array}$ & $\begin{array}{l}-\left(E_{i}^{T} P E_{j}+E_{j}^{T} P E_{i}\right) \\
E_{i}^{T} P E_{j}+E_{j}^{T} P E_{i} \\
\frac{1}{2}\left(E_{i}^{T} P E_{j}+E_{j}^{T} P E_{i}\right) \\
-\frac{1}{4}\left(E_{j}^{T} E_{i}^{T} P+P E_{i} E_{j}+E_{i}^{T} E_{j}^{T} P+P E_{j} E_{i}\right)\end{array}$ & $\begin{array}{l}-\left(E_{j}^{T} P A+A^{T} P E_{i}\right) \\
E_{i}^{T} P A+A^{T} P E_{i} \\
\frac{1}{2}\left(E_{i}^{T} P A+A^{T} P E_{i}\right) \\
-\frac{1}{4}\left(A^{T} E_{i}^{T} P+P E_{i} A+E_{i}^{T} A^{T} P+P A E_{i}\right)\end{array}$ \\
\hline
\end{tabular}

Root-Clustering in an Ellipse with Structured Uncertainties

Consider the system and perturbation matrices

$$
A=\left[\begin{array}{cc}
-0.1 & 0.6 \\
-0.4 & 1
\end{array}\right], \quad E_{1}=\left[\begin{array}{ll}
1 & 0 \\
1 & 0
\end{array}\right], \quad E_{2}=\left[\begin{array}{ll}
0 & 1 \\
0 & 1
\end{array}\right] \text {. }
$$

With $\lambda_{1}(A)=0.2, \lambda_{2}(A)=0.7$. Taking the ellipsoidal subregion with $\alpha=0.4, v=0.5, w=0.4$, then the eigenvalues $\lambda_{1}$ and $\lambda_{2}$ are located in this subregion. The bound on $\epsilon_{1}$ and $\epsilon_{2}, \mu$, can be computed directly with $Q=S=I((4.1)$ and Table I)

$$
\mu_{\epsilon, S=I}=0.0395 \text {. }
$$

Using the method proposed in [5], taking $Q=I$, we obtain the perturbation bound

$$
\mu_{e, y e d}=0.0072 \text {. }
$$

Comparing the bounds $\mu_{e, S=I}$ with $\mu_{e}$,yed we see that our bound gives an improvement of $449 \%$. A still less conservative result can be obtained by optimizing. The optimal $S$ matrix and the solution for $P(2.5)$ become

$$
S=\left[\begin{array}{rr}
0.9144 & -0.4853 \\
-0.8012 & 1.0582
\end{array}\right], \quad P=\left[\begin{array}{rr}
1.8312 & -1.6732 \\
-1.6732 & 1.8692
\end{array}\right] \text {. }
$$

The error bound then becomes

$$
\mu_{e, ~}=S_{\text {opt }}=0.1098 \text {. }
$$

Comparing the bounds $\mu_{e}, S=S_{\text {opt }}$ with $\mu_{e, S=I}$ we see that optimization gives an improvement of $178 \%$. Optimizing the $Q$ matrix and using the method of Yedavalli, the same bound as $\mu_{e, \text { yed }}$ is obtained.

The results of this example are illustrated in Fig. 1, where the location of the roots in the ellipsoidal subregion of the unit disk, with the maximum perturbation $\left(\lambda\left(A \pm \mu\left(E_{1}+E_{2}\right)\right)\right.$, are shown.

Regarding this plot, it is important to note that the roots marked with a " $x$ " are, in general, not the outermost points of a root locus determined by varying $\epsilon$ from $-\mu$ to $\mu$ in $\lambda\left(A \pm \epsilon\left(E_{1}+E_{2}\right)\right.$.

\section{General Remarks}

Remark 5.1: In remarks 3.4 and 4.1, comment is given about the optimal $S$ matrix. This $S$ matrix can be obtained with use of

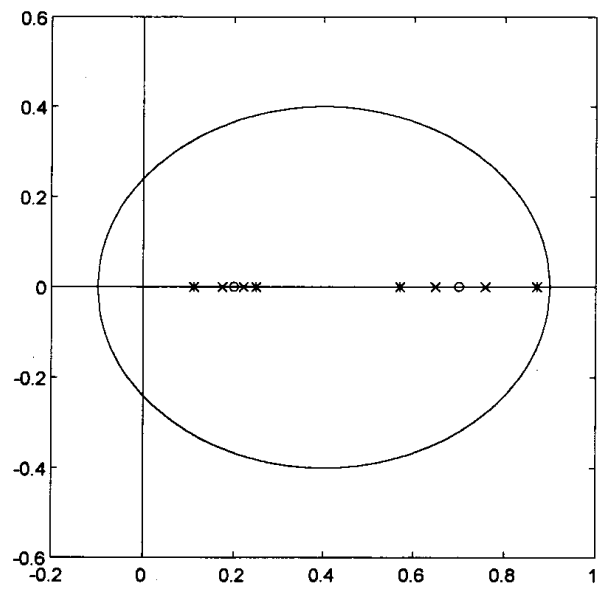

Fig. 1. Root-clustering in the ellipsoidal subregion of the unit disk. The roots of the nonperturbed system are denoted by "o", the roots of the system with the maximum perturbation bound $\mu_{e}, S=I$ are denoted by "+" and the roots with the bound $\mu_{e}, S=S_{\text {opt }}$ are denoted by " $\times$."

MATLAB $^{\mathrm{TM}}$ programs for the optimization toolbox [9]. Furthermore, note that the optimal $S$ matrix is not unique. For example, when $\hat{S}=\alpha S$ is substituted, the solution of $\hat{P}$ with (2.5) becomes (where $\hat{Q}=\alpha^{2} Q=\alpha^{2} S^{T} S$ ) $\hat{P}=\alpha^{2} P$. Clearly, the bound [(3.1) or (4.1)] obtained for $P$ and $S$ equals the bound obtained for $\hat{P}$ and $\hat{S}$.

Remark 5.2: It is important to note that the bounds derived are valid for time-invariant uncertainties. In the theorems given, however, only the location of the eigenvalues is guaranteed to remain in a specified subregion; this is true for each value of the uncertainty that satisfies the bound. So, when the bound is satisfied, at each time instant, by the time-varying uncertainties, then the eigenvalues remain in the subregion. For these time-varying uncertainties, however, this does not guarantee a certain corresponding performance. In engineering practice we are interested in performance; hence we presented bounds that guarantee, beside root-clustering in a subregion, also a corresponding performance. 


\section{CONCLUSIONS}

In this paper some improved bounds for robust performance of linear systems are obtained. Systems with structured and unstructured uncertainties are treated. For each case, optimization yields better results. For unstructured uncertainties, with shifting of the subregion and the system matrix, a less conservative result is obtained. We have presented our results for various different subregions in the form of tables. With the method proposed it is possible to determine admissible uncertainties of the system to guarantee, for time invariant uncertainties, a certain performance.

\section{APPENDIX}

Proof of Theorem 3.1: Recall the Lyapunov root-clustering criterion of Section II. From assumption (2) it follows that there exist a positive definite solution $P$ of the GLE (2.5). If the GLE of the perturbed system matrix $A+E$ has a positive definite "solution" $Q_{E}$ for that solution $P$ then the eigenvalues of the perturbed system matrix are located in the specified subregion. Thus, the eigenvalues of the perturbed system are located in $\Omega_{2}$ if

$$
\begin{aligned}
c_{00} P & +c_{10}\left((A+E)^{T} P+P(A+E)\right) \\
& +c_{11}(A+E)^{T} P(A+E)+c_{20}\left(\left((A+E)^{T}\right)^{2} P\right. \\
& \left.+P(A+E)^{2}\right)=-Q_{E}<0 .
\end{aligned}
$$

See also Yedavalli [5]. Substitution of (2.5) gives

$$
\begin{aligned}
\Leftrightarrow & c_{10}\left(E^{T} P+P E\right)+c_{11}\left(E^{T} P A+A^{T} P E+E^{T} P E\right) \\
& +c_{20}\left(\left(A^{T} E^{T}+E^{T} A^{T}+\left(E^{T}\right)^{2}\right) P\right. \\
& +c_{20} P\left(A E+E A+E^{2}\right)-Q<0 \\
\Leftrightarrow & c_{10} S^{-T}\left(E^{T} P+P E\right) S^{-1} \\
& +c_{11} S^{-T}\left(E^{T} P A+A^{T} P E+E^{T} P E\right) S^{-1} \\
& +c_{20} S^{-T}\left(\left(A^{T} E^{T}+E^{T} A^{T}+\left(E^{T}\right)^{2}\right) P S^{-1}\right. \\
& +c_{20} S^{-T} P\left(A E+E A+E^{2}\right) S^{-1}-S^{-T} S^{T} S S^{-1}<0 \\
\Leftrightarrow & c_{10} S^{-T} E^{T} P S^{-1}+c_{10} S^{-T} P E S^{-1}+c_{11} S^{-T} E^{T} P A S^{-1} \\
& +c_{11} S^{-T} A^{T} P E S^{-1}+c_{11} S^{-T} E^{T} P E S^{-1} \\
& +c_{20} S^{-T} A^{T} E^{T} P S^{-1}+c_{20} S^{-T} E^{T} A^{T} P S^{-1} \\
& +c_{20} S^{-T}\left(E^{T}\right)^{2} P S^{-1}+c_{20} S^{-T} P A E S^{-1} \\
& +c_{20} S^{-T} P E A S^{-1}+c_{20} S^{-T} P E^{2} S^{-1}-I<0 .
\end{aligned}
$$

The left side of (A.1) is a Hermitian matrix. A Hermitian matrix has the property that all the eigenvalues $\left(\lambda_{k}\right)$ are real and that it is negative definite if and only if all its eigenvalues are negative. Further, from [10], the property $\lambda_{k}(A-I)<0 \Leftrightarrow \lambda_{k}(A)-1<$ $0 \Leftarrow\|A\|-1<0$ and $\|A\|+\|B\|>\|A+B\|$, with $\|A\|=$ the spectral norm of the matrix $A$ (the largest singular value of $A$ ), gives

$$
\begin{aligned}
\Leftarrow & \left\|c_{10} S^{-T} E^{T} P S^{-1}\right\|+\left\|c_{10} S^{-T} P E S^{-1}\right\|+\left\|c_{11} S^{-T} E^{T} P A S^{-1}\right\| \\
& +\left\|c_{11} S^{-T} A P E S^{-1}\right\|+\left\|c_{11} S^{-T} E^{T} P E S^{-1}\right\| \\
& +\left\|c_{20} S^{-T} A^{T} E^{T} P S^{-1}\right\|+\left\|c_{20} S^{-T} E^{T} A^{T} P S^{-1}\right\| \\
& +\left\|c_{0} S^{-T} E^{T} E^{T} P S^{-1}\right\|+\left\|c_{20} S^{-T} P A E S^{-1}\right\| \\
& +\left\|c_{20} S^{-T} P E A S^{-1}\right\|+\left\|c_{20} S^{-T} P E^{2} S^{-1}\right\|-1<0 \\
\Leftarrow & 2\left\|c_{10} S^{-T} P\right\|\|E\|\left\|S^{-1}\right\|+2\left\|c_{11} S^{-T}\right\| E^{T}\left\|P A S^{-1}\right\| \\
& +\left\|c_{11} S^{-T}\right\|\left\|E^{T}\right\|\|P\|\|E\|\left\|S^{-1}\right\| \\
& +2\left\|c_{20} S^{-T} A^{T}\right\|\left\|E^{T}\right\|\left\|P S^{-1}\right\|+2\left\|c_{20} S^{-T}\right\|\left\|E^{T}\right\|\left\|A^{T} P S^{-1}\right\| \\
& +2\left\|c_{20} S^{-T}\right\|\left\|\left(E^{T}\right)^{2}\right\|\left\|P S^{-1}\right\|-1<0
\end{aligned}
$$

$$
\begin{gathered}
\Leftarrow\left(\left|c_{11}\right|\|P\|\left\|S^{-1}\right\|^{2}+2\left|c_{20}\right|\left\|S^{-1}\right\|\left\|P S^{-1}\right\|\right)\|E\|^{2} \\
\quad+\left(2\left|c_{10}\right|\left\|S^{-1}\right\|\left\|P S^{-1}\right\|+2\left|c_{11}\right|\left\|S^{-1}\right\|\left\|P A S^{-1}\right\|\right. \\
+2\left|c_{20}\right|\left\|S^{-T} A\right\|\left\|P S^{-1}\right\| \\
\left.+2\left|c_{20}\right|\left\|S^{-1}\right\|\left\|A^{T} P S^{-1} \mid\right\|\right)\|E\|-1<0 \\
\Leftrightarrow a\|E\|^{2}+b\|E\|-1<0
\end{gathered}
$$

with

$$
\begin{gathered}
a \equiv\left|c_{11}\right|\|P\|\left\|S^{-1}\right\|^{2}+2\left|c_{20}\right|\left\|S^{-1}\right\|\left\|P S^{-1}\right\| \\
b \equiv 2\left|c_{10}\right|\left\|S^{-1}\right\|\left\|P S^{-1}\right\|+2\left|c_{11}\right|\left\|S^{-1}\right\|\left\|P A S^{-1}\right\| \\
+2\left|c_{20}\right|\left(\left\|S^{-T} A^{T}\right\|\left\|P S^{-1}\right\|+\left\|S^{-1}\right\|\left\|A^{T} P S^{-1}\right\|\right) \\
\Leftrightarrow\|E\|<-\frac{b}{2 a}+\frac{1}{2 a} \cdot \sqrt{b^{2}+4 a} .
\end{gathered}
$$

Proof of Theorem 4.1: The first part of the proof is the same as the proof of Theorem 3.1. We continue with (A.1)

$$
\begin{aligned}
\Leftrightarrow & S^{-T}\left\{c_{10}\left(E^{T} P+P E\right)+c_{11}\left(E^{T} P A+A^{T} P E\right)\right. \\
& +c_{20}\left((E A)^{T} P+(A E)^{T} P+P(A E+E A)\right) \\
& \left.+c_{11} E^{T} P E+c_{20}\left(\left(E^{T}\right)^{2} P+P E^{2}\right)\right\} S^{-1}-I<0 .
\end{aligned}
$$

Now the substitution $E \rightarrow \sum_{i=1}^{m} \epsilon_{i} E_{i}$ is made (2.2). Abbreviating

$$
\begin{gathered}
P_{i}^{*} \equiv S^{-T} P_{i} S^{-1} ; \quad P_{i j}^{*} \equiv S^{-T} P_{i j} S^{-1} \\
P_{i} \equiv c_{10}\left(E_{i}^{T} P+P E_{i}\right)+c_{11}\left(E_{i}^{T} P A+A^{T} P E_{i}\right) \\
+c_{20}\left(\left(E_{i} A\right)^{T} P+P E_{i} A+\left(A E_{i}\right)^{T} P+P A E_{i}\right) \\
P_{i j} \equiv c_{11}\left(E_{i}^{T} P E_{j}+E_{j}^{T} P E_{i}\right) \\
+c_{20}\left(\left(E_{i} E_{j}\right)^{T} P+P E_{i} E_{j}+\left(E_{j} E_{i}\right)^{T} P+P E_{j} E_{i}\right) \\
\Leftrightarrow \sum_{i=1}^{m} \epsilon_{i} P_{i}^{*}+\sum_{i=1}^{m} \sum_{j=i}^{m} \epsilon_{i} \epsilon_{j} P_{i j}^{*}-I<0 .
\end{gathered}
$$

The left side of this inequality is a Hermitian matrix. From [10], the property

$$
\begin{aligned}
& \lambda_{k}(A-I)<0 \Leftrightarrow \lambda_{k}(A)-1<0 \Leftarrow\|A\|-1<0 \text { gives } \\
& \Leftarrow\left\|\sum_{i=1}^{m} \epsilon_{i} P_{i}^{*}+\sum_{i=1}^{m} \sum_{j=i}^{m} \epsilon_{i} \epsilon_{j} P_{i j}^{*}\right\|-1<0 \\
& \Leftarrow\left\|\sum_{i=1}^{m} \epsilon_{i} P_{i}^{*}\right\|+\left\|\sum_{i=1}^{m} \sum_{j=i}^{m} \epsilon_{i} \epsilon_{j} P_{i j}^{*}\right\|-1<0 \\
& \Leftarrow \epsilon\left\|\sum_{i-1}^{m}\left|P_{i}^{*}\right|\right\|+\epsilon^{2}\left\|\sum_{i=1}^{m} \sum_{j=i}^{m}\left|P_{i j}^{*}\right|\right\|-1<0 \\
& \Leftrightarrow a \epsilon^{2}+b \epsilon-1<0 \\
& |\epsilon|<-\frac{b}{2 a}+\frac{1}{2 a} \cdot \sqrt{b^{2}+4 a}
\end{aligned}
$$

and where $|A|=\left\{\left|a_{i j}\right|\right\}$, with $A=\left\{a_{i j}\right\}$, a $n \times n$ matrix. 


\section{REFERENCES}

[1] A. A. Abdul-Wahab, "Lyapunov bounds for root'clustering in the presence of system uncertainty," Int. J. Syst. Sci., vol. 21, no. 12, pp. 2603-2611, 1990.

[2] _ "Perturbation bounds for root-clustering of linear discrete-time systems," Int. J. Syst. Sci., vol. 22, no. 10, pp. 1775-1783, 1991.

[3] R. K. Yedavalli, "Counter-example to 'Perturbation bounds for rootclustering of linear continuous-time systems," Int. J. Syst. Sci., vol. 23 , no. 4, pp. 661-662, 1992.

[4] W. Bakker and J. S. Luo, "Comment and counter-example to 'Pertur bation bounds for root-clustering of linear discrete-time systems," Int J. Syst. Sci., vol. 24, no. 11, pp. 2205-2206, 1993.

[5] R. K. Yedavalli, "Robust root clustering for linear uncertain systems using generalized Lyapunov theory," Automatica, vol. 29, no. 1, pp. 237-240, 1993.

[6] S. Gutman and E. I. Jury, "A general theory for matrix root-clustering in subregions of the complex plane," IEEE Trans. Automat. Contr., vol. AC-26, no. 4, pp. 853-863, 1981.

[7] J. S. Luo, A. Johnson, and P. P. J. van den Bosch, "New Lyapunov robustness bounds for pole-assignment in a specified region," in Proc. IFAC World Congress, vol. 2, 1993, pp. 495-498.

[8] K. Furuta and S. B. Kim, "Pole assignment in a specified disk," IEEE Trans. Automat. Contr., vol. AC-32, no. 5, pp. 423-427, 1987.

[9] A. Grace, Optimization Toolbox: User's Guide for Use with MATLAB. South Natick, MA: The MathWorks, 1990.

[10] P. Lancaster and M. Tismenetsky, The Theory of Matrices, 2nd ed. Orlando, FL: Academic, 1985.

\section{Comments on "Strictly Positive Real Transfer Functions Revisited"}

\section{H. J. Marquez and C. J. Damaren}

Abstract - In the above paper, ${ }^{1}$ the distinction between weak and strong strictly positive real (SPR) functions was addressed, and the feedback interconnection of a weak SPR system and a passive one was shown to be stable. The purpose of this note is to show that the proof of this lemma is actually incorrect.

\section{INTRODUCTION}

The concepts of passivity and strict positive realness have been an important area of research for the last three decades. These investigations have brought a better understanding of these ideas and their applications, but also an ever increasing mismatch in the terminology adopted by different authors. The most widely accepted definitions of passivity and strict passivity are the following [1]. Define a real inner product $\langle x, y\rangle_{T}$ by

$$
\langle x, y\rangle_{T}=\int_{0}^{T} x^{T}(t) y(t) d t
$$

and let $L_{2 e}^{n}$ be the space of all functions $x: R^{+} \rightarrow R^{n}$ which satisfy $\left\|x_{T}\right\|_{2}^{2}=\langle x, x\rangle_{T}<\infty, \forall T \in R^{+}\left(R^{+}\right.$is the set of positive real numbers).

Passivity: $H: L_{2 e}^{n} \rightarrow L_{2 e}^{n}$ is said to be passive if there exists $\beta \in R$ such that

$$
\langle x, H x\rangle_{T} \geq \beta, \quad \forall x \in L_{2 e}^{n}, \quad \forall T \in R^{+} .
$$

Manuscript received January 24, 1994; revised April 11, 1994

The authors are with the Department of Engineering, Royal Roads Military College, FMO Victoria, British Columbia V0S 1B0, Canada.

IEEE Log Number 9408546.

${ }^{1}$ R. Lozano-Leal and S. Joshi, IEEE Trans. Automat. Contr., vol. 35, no. 11 , pp. 1243-1245, Nov. 1990

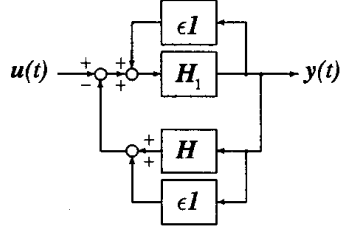

Fig. 1. The feedback system $\mathcal{S}_{\epsilon}$.

Strict Passivity: $H: L_{2 e}^{n} \rightarrow L_{2 e}^{n}$ is said to be strictly passive if there exists $\delta>0$, and $\beta \in R$ such that

$$
\langle x, H x\rangle_{T} \geq \delta\left\|x_{T}\right\|_{2}+\beta, \quad \forall x \in L_{2 e}^{n}, \quad \forall T \in R^{+} .
$$

For linear systems these definitions are closely related to the concept of strictly positive real (SPR). See the above paper ${ }^{1}$ for the definitions of weak and strong SPR. From these definitions, it is straightforward that a linear time-invariant system whose transfer function is (weak or strong) SPR is passive but, in general, not strictly passive. For example, the system $H(s)=k /(s+a), k>0$, and $a>0$ is SPR (and so passive); however, it is not strictly passive since $\operatorname{Re}[H(j \omega)] \rightarrow 0$ as $\omega \rightarrow \infty$, and therefore no $\delta$ can be found to satisfy (3). The main problem with this result is that it renders the passivity theorem nearly inapplicable for linear systems, since only biproper or improper linear systems can be strictly passive.

Motivated by this observation, the authors ${ }^{1}$ considered the class of systems which satisfy the inequality $(6 \mathrm{~b})^{1}$

$$
\frac{\int_{0}^{\infty} y^{T} u d t+\beta}{\int_{0}^{\infty} u^{T} u d t}>0
$$

where $y=H u$, and (4) is valid for all inputs $u$ such that $\|\dot{u}\|_{2} /\|u\|_{2}<\infty$. This class of systems was shown to be equivalent to those which are weak SPR and played a central role in Lemma 1. There, it was claimed that the feedback interconnection of a passive system and one that satisfies inequality (4) (see Fig. $1^{1}$ ) is stable. The intention of this note is to show that the proof of Lemma $1^{1}$ is not valid. We notice here that the condition $\|\dot{u}\|_{2} /\|u\|_{2}<\infty$ is not used in the proof of Lemma 1, and therefore in the remainder of this note it is disregarded.

Using our notation, (4) can be rewritten as

$$
\frac{\langle u, H u\rangle+\beta}{\langle u, u\rangle}>0 \text {. }
$$

A number of comments must be made concerning this definition. In the first place, it is incomplete since for expression (4) to be well defined, it is necessary for the function $u$ to belong to the space $L_{2}^{n}$. This is an important point. In fact, this issue renders incorrect the proof of Lemma $1 .^{1}$ Notice that in the proof of Lemma $1,{ }^{1}$ it is necessary to consider precisely the case where $u$ is not in $L_{2}$. Yet more appropriate is to use extended spaces and rewrite (4) as

$$
\lim _{T \rightarrow \infty} \frac{\langle u, H u\rangle_{T}+\beta}{\langle u, u\rangle_{T}}>0, \quad \forall u \in L_{2 e}^{n} .
$$

We now analyze the proof of Lemma 1 (Appendix $\mathrm{II}^{1}$ ). Since $u_{1}=-y$ and $y_{1}=u$ (refer to Fig. $1^{1}$ )

$$
\frac{\langle u, H u\rangle_{T}+\beta}{\langle u, u\rangle_{T}}+\frac{\left\langle u_{1}, H_{1} u_{1}\right\rangle_{T}+\beta_{1}}{\langle u, u\rangle_{T}}=\frac{\beta+\beta_{1}}{\langle u, u\rangle_{T}} .
$$

Here $H_{1}$ is passive, and therefore the second term on the lefthand side of (7) is greater than or equal to zero, while $H$ satisfies 